\title{
Las revistas para adolescentes como mediadoras sociales. Una aproximación al tratamiento de las drogas en 2008 y 20091
}

\section{Teenage magazines as social mediators. Studying the coverage of drugs in 2008 and 2009}

\author{
María Pilar Paricio Esteban. Universidad CEU Cardenal Herrera \\ Cristina Rodríguez Luque. Universidad CEU Cardenal Herrera \\ Pilar Sanfeliu Aguilar. Universidad CEU Cardenal Herrera \\ Francisco Núñez-Romero Olmo. Universidad CEU Cardenal Herrera
}

Recibido: 27-X-2010 - Aceptado: 22-I-2011

Resumen:

Esta investigación aborda el tratamiento de las drogas en publicaciones dirigidas a adolescentes y analiza las revistas de mayor tirada de dicha familia de soportes: Loka Magazine, Ragazza, Super Pop, Bravo por ti, Cuore y Nuevo Vale en el periodo, abril-junio de 2008, con un pilotaje de seguimiento en mayo de 2009. Utilizando como base la teoría del Framing, aplica un análisis de contenido categorial, temático y evaluativo. Se concluye que los textos no siguen las recomendaciones de la FAD para una información eficaz orientada a la prevención y adoptan una actitud neutra respecto a los efectos nocivos de las sustancias adictivas, frivolizando o proyectando como "no drogas" sustancias como el tabaco y el alcohol. Además, fomentan la "glamurización" de personajes relacionados con el consumo.

Palabras clave:

Droga; revista; prevención; adolescente; mediación social, análisis de contenido

Abstract:

This paper studies media coverage of drugs to teenagers and it analyzes the teenager's magazines with the highest rates of circulation: Loka Magazine, Ragazza, Super Pop, Bravo por ti, Cuore and Nuevo Vale about drugs in April-June 2008 and a pilot study in May 2009. Using the theory of Framing it employees a categorical, thematic and evaluative content analysis. It concludes that the articles do not follow the recommendations suggested by FAD in media coverage of drugs, they adopt a neutral attitude about their negative effects and promote the spectacle of famous people related to drugs consumption.

Keywords:

Drugs; magazine; prevention; teenager; social mediation, content analysis.

1 El presente trabajo de investigación está financiado por el proyecto subvencionado por la Fundación para el Estudio, Prevención y Asistencia a las Drogodependencias (FEPAD) de la Generalitat Valenciana a los autores en 2009. 


\section{Las drogas, los jóvenes y su reflejo en los medios de comunicación}

La Organización Mundial de la Salud, define la droga como una sustancia química que, introducida en el organismo, puede producir modificaciones de diversa índole en el funcionamiento del mismo. Esta definición podría aplicarse a cualquier medicamento, por lo que la Fundación de Ayuda contra la Drogadicción (FAD) matiza el concepto añadiendo que el problema de las drogas se refiere a un tipo de sustancias que crean dependencia, son consumidas con fines distintos a los terapéuticos y sin control médico (Fundación de Ayuda contra la Drogadicción, 1998).

El consumo de drogas entre los jóvenes se ha convertido en uno de los problemas psicosociales que más preocupa a los padres, educadores y profesionales de la salud en la actualidad. A pesar del aumento de campañas informativas sobre los riesgos y consecuencias negativas del consumo de sustancias adictivas, estupefacientes y juventud son un binomio de difícil disociación (Romero y Carcelén, 2010). Los datos son elocuentes y, aún cuando la última Encuesta Domiciliaria sobre consumo de drogas, refleja un descenso del consumo por primera vez en muchos años en nuestro país, el Informe Anual del Observatorio Europeo de Toxicomanías sitúa a España a la cabeza del continente en consumo de cocaína, éxtasis y anfetaminas, especialmente entre los jóvenes (EMCDDA, 2008; EMCDDA, 2009). A nivel internacional, el Informe Mundial sobre Drogas de la ONU (UNODC, 2008) subraya también el crecimiento en el consumo de cocaína en España en los últimos años, así como el hecho de que España se encuentra a la cabeza de la Unión Europea en consumo de esta sustancia y tan solo por detrás de Reino Unido e Italia en prevalencia de consumo (UNODC, 2010). Si atendemos a la edad de inicio, se sitúa entre los 13 y 14 años de media en nuestro país. Además, el 4\% de los adolescentes entre 15 y 16 años ha tomado cocaína alguna vez, el doble que en la mayor parte de los países europeos (EMCDDA, 2008).

En nuestros días, la mezcla de culturas, el nuevo ideal consumista de la sociedad y la pérdida de valores tienen como consecuencia la búsqueda del placer inmediato a través del consumo de drogas y, en ocasiones, su utilización como vía para escapar del malestar personal y social (FAD, 1998).

A lo largo de la historia, cada sociedad ha ido otorgando distintos significados al concepto droga, lo que denota su carácter puramente social. En este sentido, Javier Arza señala que el fenómeno del consumo no se explica fuera de un contexto (Arza, 2002: 16)². En la actualidad, se está extendiendo el consumo de drogas asociado al ocio, lo que da lugar a una nueva cultura de la diversión vinculada al abuso de sustancias adic-

\footnotetext{
"La droga, sin que un individuo la consuma de una manera determinada, sin que una cultura concreta le aporte sentido y significado, sin un contexto (histórico, físico, sociocultural, político y económico) en el que se produce el consumo, no deja de ser una sustancia aislada que, por sí misma, no explica el fenómeno del consumo de drogas".
} 
tivas. Incluso, en algunos ambientes el consumo de drogas se transmite como algo "seguro, aceptable, prestigioso e incluso beneficioso para la consecución de logros sociales y para la satisfacción de las necesidades personales" (Calafat y PND., 2004: 107).

Un factor esencial en la prevención y asistencia de las drogodependencias es la información orientada a la educación en la prevención. En este sentido, las instituciones que trabajan en materia de prevención dedican un gran esfuerzo a los programas y campañas para tratar de lograr la mayor eficacia de sus mensajes en las audiencias clave y, en particular, en los grupos que se inician en las drogas. El público necesita información sobre los efectos y consecuencias de las drogas para poder tomar decisiones racionales y una comunicación efectiva es importante para que esas decisiones se tomen correctamente (Sitthi-amorn y Ngamvithayapongse, 1998).

Los medios de comunicación social no son solo transmisores de información, sino que también son un poderoso medio de acción para el desarrollo integral de los individuos (Vega Fuente, 2004). Si consideramos que "el periodismo es un saber prudencial que consiste en la comunicación adecuada del saber sobre las realidades humanas actuales que a los ciudadanos les es útil saber para actuar libre y solidariamente" (Galdón, 1999: 25), en la problemática de las drogas, el periodista tiene una función social, y si, además, hablamos de Periodismo de servicio, del medio por responsabilidad social buscaría dar una respuesta eficaz a las necesidades reales de los usuarios de la información, teniendo en cuenta el interés creciente del lector por temas propios que le afectan en lo personal (Diezandino, 1994). Así, en relación al tratamiento informativo de las drogas, es importante elaborar una información contrastada y de calidad, con estrecha colaboración entre expertos en drogodependencias e informadores, empresas y colectivos que trabajan en prevención para formar profesionales especializados necesarios en un tema de tal calado social (Del Pueyo, 2004).

De hecho, la FAD recomienda a los informadores mantener una actitud responsable y activa en el fomento de la promoción de valores educativos, formativos y preventivos (no cabe la neutralidad ni la asepsia ante los efectos nocivos de las drogas, tanto las legales como las ilegales). También demanda extremar la precaución en la contextualización y evitar el trato de favor que se dispensa, por inercia o desconocimiento, a determinadas drogas (lúdicas), como las de síntesis, presentadas como inocuas. Con respecto a las asociaciones de términos o ideas, desde la FAD indican adecuado evitar las combinaciones inadaptados-drogas, marginados-drogas, rebeldes-drogas, jóvenes-drogas y clases dirigentes-drogas, ya que se estaría legitimando el consumo en virtud de la pertenencia a un estrato, condición o categoría social. Igualmente consideran idóneo informar de los efectos negativos de las drogas, pero también de las actividades positi- 
vas que instituciones y ciudadanos realizan para frenar su consumo. En este sentido, aluden a analizar los hechos desde todos los puntos de vista, no solo el exclusivamente judicial y/o policial para que no prime la visión del drogadicto delincuente. Con respecto a los datos, desde la FAD recomiendan que aunque las cifras señalen una disminución de la percepción del riesgo del consumo de sustancias adictivas y un descenso en la edad de inicio en determinados consumos, los periodistas no pueden generar una imagen alarmista que afecte de manera global a la juventud. La mayoría de los jóvenes no son consumidores de drogas (Fundación de Ayuda contra la Drogadicción, 2008: 18-21).

En este sentido, los medios han de aceptar su responsabilidad y transmitir una información contrastada, veraz y elaborada desde una perspectiva multidisciplinar, en la que el concepto de droga vaya más allá de la legalidad de cada sustancia o de su procedencia, mencionando las causas y consecuencias que implica el consumo de drogas con el fin de ofrecer un enfoque plural y no moralista que promueva el debate y fomente un espíritu crítico y responsable (López de Luzuriaga y Bermejo, 2004).

Aunque los jóvenes hacen un consumo de los medios de comunicación que se orienta fundamentalmente hacia el entretenimiento, no desprecian su uso como fuente de información (Martínez Verdú, 2007). De hecho, los medios poseen un potencial muy importante para desarrollar políticas y actuaciones en materia de drogodependencias y, por su poder de influencia en el público, pueden desempeñar también un gran papel en la educación y prevención (Pantoja Vargas y Abeijón, 2004). Es más, tienen un rol muy importante al elevar asuntos a la agenda e incrementar sus oportunidades de recibir consideración en agendas institucionales. Además, ciertos personajes en los medios pueden actuar como líderes de opinión dando publicidad a un asunto concreto o particular (Cobb y Elder, 1971).

Sin embargo, la agenda temática de los medios tiene una fuerte influencia del trabajo de las fuentes (Berkowitz, 1992; Curtin, 1999; Driedger, 2008; Lowry y Xie, 2007) y, en especial, de los gabientes de comunicación (Bauer y Gaskell, 2002; Kiousis, Popescu, y Mitrook, 2007; Trammell y Brown, 2007; Turk, 1986). Varios estudios de agenda building demuestran que los periodistas dan credibilidad a las fuentes proporcionadas por agentes de departamentos de comunicación, que influyen entre un 44 y un 80 por ciento en el contenido de las noticias (Curtin, 1999; Shallot y Johnson, 2006).

En el presente análisis nos centraremos en el papel de las revistas para adolescentes en la prevención de las drogodependencias, prestando especial atención al encuadre o frame que los periodistas dan a los contenidos. Tomaremos como base la teoría del Encuadre o frame, una teoría de efectos de los medios según la cual los actores o las fuentes impactan a partir del frame setting en cómo se configuran los encuadres de los medios, salvando, claro, las diferencias entre frame y segundo nivel de agenda-setting (Gamson, W.A. y Mo- 
digliani, A., 1989; Scheufele, 1999). Tal y como clarifica en la evolución de sus teorías McCombs, el segundo nivel de agenda se refiere a atributos, y los encuadres son atributos muy especiales: "Attributes defining a central themes are frames" (McCombs, 2005)3. En este sentido, nos centraremos en los encuadres utilizados por los periodistas como ideas vertebradoras de la pieza periodística siguiendo a Entman (1993: 52) .

Las investigaciones que abordan el tratamiento de las drogas en los medios de comunicación no contemplan como objeto de estudio publicaciones similares a las analizadas, centrándose más en prensa de información general. En tales trabajos se pone de manifiesto que los medios generalistas, cuando informan sobre las drogas, lo hacen en clave de información de sucesos o de tribunales y pocas veces lo hacen teniendo en cuenta su función formativa y/o educativa. Además, estos estudios insisten en la gran oportunidad que ofrecen los medios como plataformas o canales para la difusión de las campañas de prevención y sensibilización frente a la drogadicción y, por tanto, sus grandes posibilidades como instrumentos para aumentar la percepción del riesgo para la salud que supone el consumo de drogas (Berrio, 2000; Colectivo Abierto de Sociología, 1997; Costa y Pérez, 1987; Costa y Pérez, 1989; Del Río, 1991; Fernández-Cid, 1996; Ferrer y Dacosta, 1994; García Nebreda, 1987; Martín Nieto, 1998; Ministerio de Sanidad, 2000; Oliva, 1986; Pantoja Vargas y Abeijón, 2004; Paricio Esteban, Sanfeliu Aguilar, y Sanfeliu Montoro, 2002; Partenen y Montoenm, 1989; Prieto, 1987; Quintá, 1989; Rekalde, 2002; Solans, 1991; Vega Fuente, 1995).

\section{Metodología, hipótesis y objetivos}

Teniendo en cuenta estos antecedentes, el objetivo de la presente investigación es doble: Por un lado, estudiar el tratamiento informativo de las drogodependencias en los medios de comunicación impresos dirigidos a jóvenes y adolescentes; y, por otro, verificar el grado de sensibilización de los medios objetos de estudio, en la prevención de las drogodependencias.

Siendo la drogadicción un problema de primer orden en el caso español, como demuestran los estudios realizados por diversas entidades (EMCDDA 2008; EMCDDA, 2009; UNODC, 2008; UNODC, 2010), entendemos que la información sobre drogas se orientará a prevenir el consumo de drogas y tendrá un peso

\footnotetext{
"Los atributos que definen un tema central son encuadres".

4 "To frame is to select some aspect of a perceived reality and make them more salient in a communicating text in such a way as to promote a particular problem definition, causal interpretation, moral evaluation, and for treatment recommendation for the item described". "Enmarcar es seleccionar algunos aspectos de una realidad percibida y hacerlos destacar en un texto comunicativo, de tal manera que promueva la definición particular de un problema, una interpretación causal, una evaluación moral y una recomendación sobre el mismo". Traducción de los autores.
} 
considerable en términos de espacio y de intensidad en el tratamiento informativo en los medios de comunicación dirigidos especialmente a jóvenes y adolescentes. Así formulamos la primera hipótesis:

H1: Constituyendo la drogadicción en España un problema de primer orden y dada la edad media de inicio en el consumo, cabe esperar un peso importante de esta temática en la prensa dirigida a adolescentes con una especial atención a las informaciones sobre temáticas relativas a la prevención del consumo de drogas en el público diana.

Teniendo en cuenta las recomendaciones de la FAD sobre la información sobre drogas en las que se sugiere a los informadores una actitud responsable y activa para promover la prevención y los valores educativos, en los que no cabe la asepsia sobre los efectos nocivos de las drogas y es importante la información sobre actividades positivas que desarrollan instituciones y ciudadanos para prevenir, formulamos nuestra segunda hipótesis.

H2: En las revistas dirigidas a adolescentes se prevé hallar una actitud no neutral frente a los efectos nocivos de la drogadicción, con un contexto y encuadre o frame de las informaciones que ayude a fomentar valores educativos y preventivos, potenciando los ejemplos de actividades positivas desarrolladas por instituciones y ciudadanos.

Considerando estrictamente las publicaciones dirigidas a adolescentes, resultan también de interés las recomendaciones del Servicio de Información del Departamento norteamericano sobre drogas Substance Abuse and Mental Health Service Administration, que recomienda no utilizar personas alcohólicas como modelos, más aún si son famosos, al tratar los problemas relacionados con el alcohol y otras drogas dirigiéndose a jóvenes (Plan Nacional sobre Drogas, 2000). Además, aconsejan evitar participar en la "glamurización" o glorificación de los profesionales de los medios y del mundo del espectáculo como grandes fumadores o bebedores, destacando, en su lugar, aquellos que no realizan estas prácticas e informando de las pérdidas laborales y de salud que el consumo de estas drogas puede propiciar. En este sentido, formulamos la tercera hipótesis.

H3: En las revistas dirigidas a adolescentes se prevé encontrar una especial atención a personajes famosos, ídolos juveniles, pero evitando relacionarlos con el consumo de sustancias abusivas o estupefacientes con el fin de que actúen como mediadores sociales en la prevención de las drogodependencias.

A partir de dichas premisas y con el objetivo de conocer el tratamiento informativo las drogas en las revistas juveniles que nos ayude a validar o refutar las hipótesis de partida, hemos realizado un análisis aplicando una metodología cuantitativa y cualitativa. En este sentido, hemos utilizado el método de medición de la exposición al mensaje, que comprende el análisis de la cobertura mediática e impacto y el análisis del 
contenido de las informaciones. Siguiendo a Bardin (1986), hemos utilizado dos tipos de análisis de contenido: análisis de contenido de tipo categorial (análisis temático y de sustancias) y de tipo evaluativo del encuadre de la información. En el análisis categorial, las categorías temáticas se han diseñado ad hoc y son las siguientes:

A) Campañas y actuaciones en materia de asistencia y prevención

-Lanzamiento de Campaña.

-Actividades de Campaña

-Resultados de Campaña

a) Nivel de participación

b) Reducción de consumo

c) Otros

B) Consumo de drogas

-Consumo de una droga en particular (FEPAD)

- Alcohol

- Opiáceos

- Hipnóticos y tranquilizantes

- $\mathrm{GHB}$

- Xantinas y Tabaco

- Anfetaminas

- Cocaína

- LSD

- Hongos alucinógenos

- Ketamina

- Cannabis

- Inhalantes

- Drogas de Síntesis

- Cristal

- Esteroides/ Anabolizantes.

- Consumo en general
- Consumo por géneros

- Consumo por edad

- Consumo por otros criterios

C) Instituciones que trabajan en prevención y asistencia a la drogadicción

D) Personajes protagonistas en la información

- Personajes famosos relacionados con las drogas (con especial atención a los ídolos juveniles)

- Otros personajes

- Consumidos anónimo

- Traficante

- Familia del consumidor

- Personal sanitario

- Personal policial y judicial

- Otros

E) Causas vinculadas a la drogadicción

H) Consecuencias de la drogadicción

F) Aspectos psicosociales de la drogadicción

G) Aspectos judiciales vinculados a la drogadicción

I) Ocio y programas dirigidos a jóvenes (series de TV...) vinculados con el tema

J) Otros aspectos relativos a las drogodependencias 
Hay que explicar que, en el caso de los personajes relacionados con las drogas, se han contabilizado las referencias explícitas en las que aparecen asociados al consumo y las implícitas, es decir, aquellas en las que los personajes simplemente figuran por su papel en la ficción o las imágenes publicadas junto a los textos en las que aparecen en situaciones de consumo. Como advierte la FAD, todas ellas pueden contribuir a la glorificación de los personajes como fumadores, bebedores o consumidores de drogas.

Por otra parte, el análisis evaluativo comprende el encuadre y la dirección. El análisis del encuadre nos permitirá verificar el contexto en el que los medios enmarcan las informaciones sobre drogodependencias y consideraremos la intensidad con la que tratan las drogas, de lo que se puede concluir la importancia concedida al tema y el grado de sensibilidad de la cabecera con el mismo (Entman, 1993; Gamson y Lash, 1983; Gamson, W. A. y Modigliani, A., 1989; Goffman, 1974; Goffman, 1981; Sádaba, 2001; Scheufele, 1999). El diseño de las variables de estudio del análisis evaluativo de la intensidad o encuadre se ha fundamentado en la metodología de análisis (López Aranguren, 1989) utilizada en la Tesis Doctoral defendida en 1998 por Paricio Esteban (1999) y en las tipologías de análisis definidas por Bardin (1986). Las variables establecidas son:

A. Ubicación de la noticia:

- Emplazamiento (página): Portada; Portada y desarrollo interior, contraportada, varias páginas, Par e Impar.

B. Forma de aparición: Todo; Incrustación más foto; Incrustación; Referencia; Referencia más foto, solo foto.

C. Género periodístico: Informativo; Interpretativo; Opinión.

D. Acompañamiento o recursos gráficos para destacar la noticia: Varias fotos y recursos; varias fotos, una foto, con recursos, Sin acompañamiento.

Para estudiar en detalle los contenidos publicados en las cabeceras analizadas, se ha desarrollado una serie de 22 identificadores del encuadre a raíz del análisis pormenorizado en el que se ha contabilizado el número de páginas que ocupa cada frame. Las categorías han sido creadas ad hoc y consensuadas por el equipo investigador tras un estudio preliminar de las publicaciones elegidas y tras una fase de pre análisis. 


\section{Música}

Textos centrados en la actualidad musical, conciertos, eventos, festivales o cantantes

\section{Cine}

Textos centrados en el mundo del cine, vidas de actores, estrenos, rodajes, etc.

\section{Televisión}

La televisión aparece como encuadre fundamental de los textos, programación, fichajes televisivos, programas, etc.

\section{Sexo}

Relaciones sexuales, y todo lo relacionado con ello

\section{Test/Consultorio/Interactividad}

Test para el público, sección de cartas al director, secciones de interactividad en las que los lectores participan o envían material a la revista.

\section{Moda}

Textos que hablan de desfiles, modelos, diseñadores, pasarelas.

\section{Belleza}

Textos que versan fundamentalmente sobre cuidados de belleza femenina, tratamientos faciales, corporales, maquillajes, peluquería, etc.

\section{Famosos}

Lo central son los personajes conocidos por sus relaciones sentimentales o por su posición social en el mundo del corazón en género noticioso

\section{Tecnología}

El tema esencial son avances de la tecnología, internet, redes sociales, dispositivos electrónicos

\section{Deportes}

Mundo deportivo y de competiciones

Agenda

Propuestas en avance en un determinado lugar, enmarcado como tal. Conciertos, exposiciones, obras teatrales, películas.

\section{Ocio}

Textos dedicados al tiempo libre y cómo emplearlo con propuestas.

\section{Psicología}

Textos dedicados a temas relacionados con la psique humana, sentimientos, emociones, situaciones personales, etc.

\section{Humor}

Textos dedicados al humor, ya sea gráfico en viñetas o a modo de textos humorísticos.

\section{Otros}

Se incluyen por ejemplo aquí sorteos, posters, horóscopos, noticias y reportajes con estilo informativo clásico, páginas dedicadas a publicidad y otro tipo de contenidos 
El análisis de la dirección comprende el estudio del tono de la noticia estableciendo las categorías neutro (no contienen ninguna valoración presentando los hechos de forma objetiva), a favor (contiene valoraciones favorables), en contra (incluye valoraciones negativas) y frivoliza (se utiliza un tono irónico o superficial en el tratamiento informativo del tema).

El corpus de la investigación está integrado por las informaciones sobre el tema en las publicaciones especialmente afines al público diana en materia de prevención, las conocidas popularmente como revistas de adolescentes por que su público objetivo está comprendido entre los 12 y 17 años. En este sentido, entendemos que para las campañas de prevención de las drogodependencias es una familia de soportes de especial interés, por situarse en dicho target la media de edad de inicio en el consumo de sustancias adictivas. Por ello, este medio es especialmente idóneo para alcanzar al público que se inicia en las drogas y tratar de disuadirle. Siguiendo los datos de la OJD y del EGM, del total de las revistas dirigidas a adolescentes se han seleccionado seis publicaciones de mayor tirada y difusión que son:

Tabla I. Periodicidad e índice de lectura de las cabeceras estudiadas

\begin{tabular}{|lcc|}
\hline Cabecera & Periodicidad & Índice de lectura EGM \\
\hline Loka Magazine & Mensual & 415.000 \\
\hline Ragazza & Mensual & \\
\hline Super Pop & Quincenal & 608.000 \\
\hline Bravo por ti & Quincenal & 525.000 \\
\hline Cuore & Semanal & 767.000 \\
\hline Nuevo Vale & Semanal & 413.000 \\
\hline
\end{tabular}

Fuente: Elaboración Propia

El estudio se centra en el periodo de abril a junio de 2008 de forma intencional ya que en dicho periodo encontramos meses en los que se lanzaron campañas institucionales de prevención de drogas lo que nos permitirá verificar su tratamiento y fechas en las que a priori no hay ninguna iniciativa preventiva que con-

\footnotetext{
Resumen General del EGM, año móvil abril 2008 a marzo de 2009. En el caso de Ragazza, no hay datos en el EGM ni los tiene la dirección de la publicación. Según el EGM, el diario de información general más leído, El País, tiene un índice de 2.081.000 lectores/día, seguido de El Mundo, con 1.309.000 lectores/día. Si pasamos a cabeceras regionales, en la Comunidad Valenciana, los dos diarios regionales más importantes, Levante y Las Provincias, tienen índices de 274.000 lectores/día y 167.000 lectores/día respectivamente. La revista más leída, Cuore, tiene un índice de lectura 2,95 veces superior al de Levante y 4,84 veces superior al de Las Provincias.
} 
dicione la información publicada ${ }^{6}$. Además, se ha realizado un pilotaje de seguimiento en 2009 (concretamente del mayo de 2009) para observar posibles cambios en el tratamiento y en los modelos informativos encontrados en el periodo de 2008. Para la recogida de información, se ha realizado una labor hemerográfica consultando todos los números. Por otro lado, se ha realizado un estudio de hemerografía descriptiva sobre tres ejemplares aleatorios en 2009 como base para establecer la proporción de la información relacionada con drogas en relación con el total de la información publicada.

\section{Resultados y discusión}

Los tres encuadres con más presencia en cuanto a cantidad de páginas ocupadas en las cabeceras estudiadas son, en primer lugar, la categoría Otros, que incluye publicidad, noticias, horóscopos, concursos, etc., y después, Famosos y Moda, con un porcentaje elevado. Los temas Televisión, Consultorio/Interactividad y Belleza también reciben una cobertura destacable en las revistas estudiadas.

Figura 1. Promedio de páginas dedicadas a cada frame o encuadre.

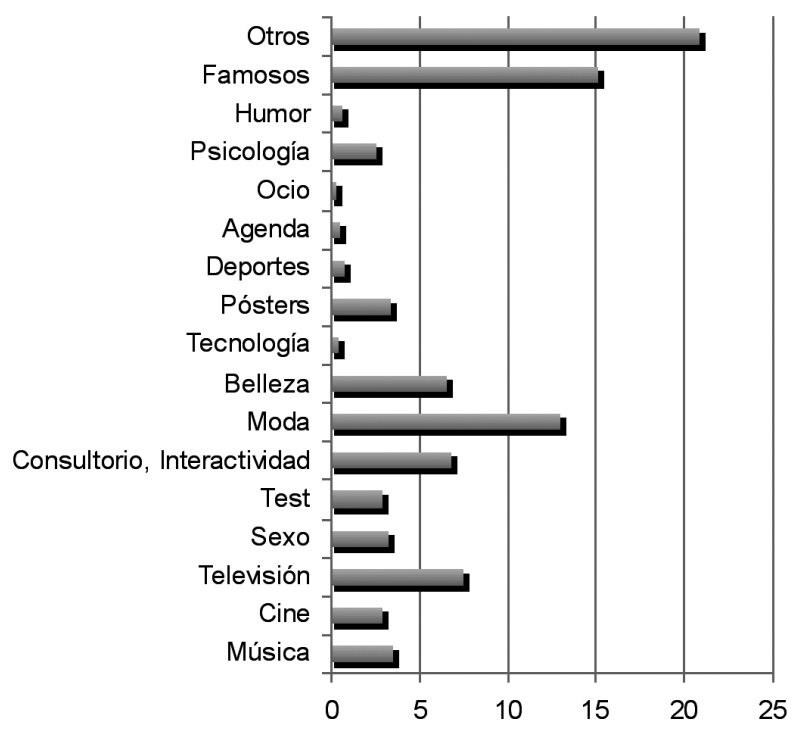

Fuente: Elaboración Propia

6 La FAD, por ejemplo, lanza una de sus campañas en mayo de 2008. 
En el estudio descriptivo también se han analizado los personajes sobre los que informan las distintas cabeceras objeto de estudio para poder comparar estos resultados con los de los personajes que aparecen relacionados con las drogas. Los personajes que más veces figuran en los ejemplares estudiados son los actores Martín Rivas, Maxi Iglesias y el grupo Jonas Brother. Por categorías, los pertenecientes a la categoría TV son los que más veces aparecen por ejemplar, seguidos por los personajes de Música. Sin embargo, los deportistas son los personajes que tienen menos referencias por ejemplar.

Tabla II. Personajes más referenciados por categorías.

\begin{tabular}{|lcc|}
\hline Temática & Personaje & Frecuencia \\
\hline Cine & Brad Pitt & 17 \\
& Lindsay Lohan & 15 \\
& Jennifer Lopez & 14 \\
\hline Deportes & Cristiano Ronaldo & 15 \\
& Fernando Torres & 12 \\
& Rafa Nadal & 10 \\
\hline Moda & Kate Moss & 15 \\
& Siena Miller & 12 \\
\hline Música & Jonas Brothers & 30 \\
& Rihanna & 23 \\
& Britney Spears & 18 \\
\hline Personajes & Paris Hilton & 14 \\
& Nicole Richie & 6 \\
& Darek & 4 \\
\hline Televisión & Maxi Iglesias & 36 \\
& Martín Rivas & 36 \\
& Miguel Á. Silvestre & 28 \\
\hline
\end{tabular}

Fuente: Elaboración Propia

\subsection{Estudio del tratamiento informativo de las drogas}

En primer lugar, es destacable que en el período analizado de 2008 hallamos 60 informaciones con referencias a las drogas y en el pilotaje de 2009 un total de 40, con lo que, en proporción, en 2009, centrándonos 
solo en un mes, hay más atención de los medios estudiados al tema de las drogas. Llama la atención, sin embargo, que en ninguno de los dos períodos analizados se encuentren campañas sobre prevención de drogodependencias o ejemplos de acciones positivas relacionadas con la prevención del consumo de sustancias adictivas.

Por otra parte, la revista con más artículos con referencias del tema es Cuore, tanto en el periodo abril-junio de 2008 como en mayo de 2009. De hecho, en el primer periodo, Cuore publicó casi el 54\% de los textos hallados con referencias a las drogas y, en el segundo periodo, casi el 60\%. En el primer periodo, esta revista publicó muchos más artículos sin referencia explícita a las drogas y, sin embargo, en el segundo periodo, el número de artículos con y sin referencia explícita al tema es similar. Super Pop es la revista que publicó menos artículos con referencias a las drogas en los periodos estudiados.

Tabla III. Artículos con referencias a drogas por cabeceras.

\begin{tabular}{|lcc|}
\hline Revistas & Abril-junio 2008 & Mayo 2009 \\
\hline Bravo por ti & 0 & 1 \\
\hline Cuore & 32 & 21 \\
\hline Loka Magazine & 8 & 5 \\
\hline Nuevo Vale & 17 & 11 \\
\hline Ragazza & 3 & 1 \\
\hline Super Pop & 0 & 40 \\
\hline Totales & 60 & 1 \\
\hline
\end{tabular}

Fuente: Elaboración Propia

En el análisis realizado en 2008, llama la atención el reducido número de informaciones que tratan el fenómeno de las drogas (226 de las cuales 60 son con referencias explícitas al fenómeno y 166 con referencias implícitas). En el estudio de seguimiento de 2009, seguimos encontrando un número reducido de informaciones (67 de las cuales son 40 con referencia explícita al tema y 27 con referencias implícitas). No obstante, en proporción hay un mayor número de ellas teniendo en cuenta que el pilotaje se hace sobre un mes frente a tres. Entendemos que dada la problemática de inicio de consumo en edades tempranas -público objetivo de la revista-, el medio debería tener una mayor sensibilidad con el tema destinando un mayor espacio a su tratamiento. 
Si se analizan los artículos con referencias a las drogas en las revistas objeto de estudio y se clasifican según la temática, podemos observar que la gran mayoría de los textos se refiere a famosos (95,6\% en el periodo abril-junio de 2008). La revista Cuore fue la que publicó la mayor parte de estos artículos sobre personajes (53\% del total).

El consumo de drogas es un tema poco tratado en las cabeceras estudiadas en el periodo abril-junio de 2008 (6,7\%), centrándose en los personajes en un 83,05\% de los casos. En mayo de 2009, los resultados son similares. La temática Personajes agrupa la mayoría de los artículos publicados con referencias a las drogas con el 65\% del total. La revista Cuore es también la que más textos publica sobre personajes (40 textos con referencias explícitas e implícitas) en este periodo. Por otra parte, el consumo de drogas aparece en más textos (15\%) y la revista Nuevo Vale sigue siendo la que más artículos publicó sobre consumo de drogas (60\%), manteniendo este porcentaje similar al del periodo abril-junio de 2008 (50\%).

En el análisis realizado en 2008, algunas revistas realizan un tratamiento importante de las series de TV donde el problema de las drogas forma parte de la trama, idealizando a protagonistas situados en el lado negativo del problema (traficantes, por ejemplo). En el estudio de seguimiento de 2009, baja el espacio dedicado a la información sobre series de TV (4 textos), en parte motivado por el menor tratamiento de la serie "Sin tetas no hay paraíso" al desaparecer la figura del Duque, que inspiraba secciones enteras de algunas revistas analizadas (en 2008 encontramos 6 informaciones centradas en ese personaje lo que representa el $100 \%$ de las informaciones sobre Televisión relacionadas con drogas y un 13\% de las informaciones sobre personajes en dicho período).

\subsubsection{Los personajes en la información sobre drogas}

Si analizamos los personajes que más aparecen en las noticias que tratan el tema de las drogas en abriljunio de 2008, destacan dos figuras de manera importante: Amy Winehouse (19\%) y Paris Hilton (14,5\%). En el caso de Amy Winehouse, los artículos con referencias implícitas a las drogas son un 68,5\% y en el caso de Paris Hilton, el 100\% son con referencias implícitas. 
Tabla IV. Personajes relacionados con las drogas en los artículos en abril-junio de 2008.

\begin{tabular}{|lccc|}
\hline Personajes & Sin referencia & Con referencia & Totales \\
\hline Amy Winehouse & 28 & 13 & 41 \\
\hline Otros & 23 & 11 & 34 \\
\hline Paris Hilton & 31 & 0 & 31 \\
\hline Rihanna & 18 & 0 & 18 \\
\hline K. Moss & 12 & 6 & 18 \\
\hline Brithey Spears & 13 & 4 & 17 \\
\hline M. Barton & 11 & 2 & 13 \\
\hline Olsen & 10 & 0 & 10 \\
\hline Lindsay Lohan & 5 & 5 & 10 \\
\hline Dorothy Perkins & 4 & 4 & 8 \\
\hline Naomi Campbell & 5 & 2 & 7 \\
\hline Duque & 4 & 2 & 6 \\
\hline J. Timberland & 3 & 0 & 3 \\
\hline Totales & 167 & 49 & 216 \\
\hline
\end{tabular}

Fuente: Elaboración Propia

Si nos fijamos en mayo de 2009, Rihanna es la que más veces aparece en artículos donde se trata el fenómeno de las drogodependencias (17\%), seguida por Amy Winehouse (13\%) y por Paris Hilton (7,5\%). Los tres primeros personajes de mayo de 2009 son los mismos que de abril-junio de 2008, solo que en orden distinto. Además, los porcentajes de los artículos en los que aparecen estos tres personajes en mayo de 2009 comparados con el periodo anterior son bastante similares. Llama la atención, sin embargo, que Amy Winehouse aparezca en más artículos con referencia explícita a las drogas que sin ella en el periodo mayo 2009, lo que da una idea de la relación tan estrecha que existe entre esta cantante y el consumo de estupefacientes. 
Tabla V. Personajes relacionados con las drogas en los artículos en mayo de 2009.

\begin{tabular}{|lccc|}
\hline Personajes & Sin Referencia & Con Referencia & Totales \\
\hline Rihanna & 9 & 0 & 9 \\
\hline Amy Winehouse & 2 & 5 & 7 \\
\hline P. Hilton & 4 & 0 & 4 \\
\hline K. Moss & 2 & 2 & 4 \\
\hline Lindsay & 3 & 0 & 3 \\
\hline M. Barton & 3 & 0 & 3 \\
\hline Angry & 2 & 0 & 2 \\
\hline Briyhey & 0 & 2 & 2 \\
\hline Enimen & 0 & 2 & 2 \\
\hline M.Rouke & 0 & 2 & 2 \\
\hline Otros & 2 & 13 & 15 \\
\hline Totales & 27 & 26 & 53 \\
\hline
\end{tabular}

Fuente: Elaboración Propia

En 2009, los personajes que más veces aparecen en las publicaciones analizadas en los ámbitos de Moda (Kate Moss), Cine (Lindsay Lohan) y Famosos (Paris Hilton) también se relacionan de manera significativa con el consumo de drogas. En la cobertura de las drogas en las revistas, hemos encontrado un número elevado de (18) donde se presenta como modelo para el adolescente un personaje con problemas de adicción que, además, defiende como un estilo de vida.

Por ejemplo, en 2008 (ejemplar número 100) encontramos un texto de una lectora dedicado a Amy Winehouse, titulado "La Apoteosis de Amy Winehouse". El texto incluye un poema de un libro de la lectora titulado Una drogata deliciosa, siendo un fragmento del mismo: "eres la flor más deliciosa de la vida y si para ti la semilla de su flor es la cocaína, bendita seas ¡ole tus ovarios! ¡Has tirado por tierra en una calada de pitillo cantada, toda esa falsa propaganda contra la drogadicción!”

\subsubsection{Resultados por sustancias}

También hemos realizado un estudio del tratamiento de las sustancias en las informaciones. En el periodo abril-junio de 2008, sólo un 25\% de los artículos estudiados tenía referencias a alguna sustancia. De ellos, 
la mayoría hacen referencia a las drogas en general (43\%), al alcohol (28,5\%) y al tabaco (18\%). Además, solo en Cuore y Loka aparecen referencias a cocaína, marihuana o éxtasis, pero en proporciones muy bajas. En el periodo mayo de 2009, el 76\% de los artículos tienen referencias a sustancias. De ellos, la mayoría son sobre alcohol y tabaco (61\%) y sobre drogas en general (21,5\%). Solo Cuore, Loka y Nuevo Vale publicaron artículos con referencias a la cocaína, la marihuana y la heroína, pero con unos porcentajes muy bajos.

\subsubsection{Resultados sobre frames de los textos}

Si hacemos referencia a los encuadres de los textos analizados, en el periodo abril-junio de 2008, la mayoría de los artículos trata sobre famosos, seguidos de Moda y Otros.

Tabla VI. Artículos sobre drogas según encuadres en abril-junio de 2008

\begin{tabular}{|lcccccccc|}
\hline Revistas & Famosos & Moda & Interactividad & Belleza & TV & Música & Cine & Otros \\
\hline Bravo por ti & 7 & 1 & 0 & 0 & 0 & 0 & 0 & 8 \\
\hline Cuore & 55 & 10 & 3 & 1 & 3 & 0 & 0 & 50 \\
\hline Loka & 5 & 1 & 2 & 0 & 1 & 0 & 0 & 5 \\
\hline Nuevo Vale & 22 & 0 & 1 & 1 & 4 & 7 & 1 & 15 \\
\hline Ragazza & 0 & 4 & 2 & 3 & 1 & 1 & 0 & 2 \\
\hline Super Pop & 11 & 0 & 0 & 0 & 0 & 0 & 0 & 5 \\
\hline Totales & 100 & 16 & 8 & 6 & 9 & 8 & 1 & 85 \\
\hline
\end{tabular}

Fuente: Elaboración Propia

En el periodo mayo de 2009, el encuadre dedicado a los famosos continúa siendo el mayoritario, seguido del encuadre Otros y Moda con 8 textos cada uno (se mantiene asi el encuadre mayoritario del estudio realizado en 2008). 
Tabla VII. Artículos sobre drogas según encuadres en mayo de 2009.

\begin{tabular}{|lcccccccc|}
\hline Revistas & Famosos & Moda & Interactividad & Belleza & TV & Música & Cine & Otros \\
\hline Bravo por ti & 1 & 0 & 0 & 0 & 0 & 0 & 0 & 0 \\
\hline Cuore & 26 & 6 & 1 & 2 & 1 & 0 & 0 & 4 \\
\hline Loka & 1 & 0 & 0 & 0 & 0 & 1 & 1 & 2 \\
\hline Nuevo Vale & 8 & 0 & 2 & 0 & 2 & 0 & 0 & 2 \\
\hline Ragazza & 1 & 2 & 0 & 1 & 0 & 0 & 0 & 0 \\
\hline Super Pop & 1 & 0 & 0 & 0 & 0 & 0 & 0 & 0 \\
\hline Totales & 37 & 8 & 3 & 3 & 3 & 1 & 1 & 8 \\
\hline
\end{tabular}

Fuente: Elaboración Propia

Este encuadre con el que se tratan las drogodependencias contribuye a banalizar el problema pues el tema se vertebra entorno al mundo de los famosos, noticias sobre ellos, entrevistas sobre ellos o con el mundo de la moda. Este tratamiento, además, favorece la asociación del problema al ídolo juvenil contribuyendo también a la asociación de éxito con drogadicción.

En cuanto a las páginas en las que se ubicaron los artículos analizados, en el periodo abril-junio de 2008, la mayoría lo hicieron en página par (61,5\%). Esta ubicación mayoritaria significa que los artículos analizados se ubican en las páginas poco destacadas dentro de la publicación. Además, solo el 2,5\% de los artículos aparecieron en la portada, la página más destacada de la publicación. En el periodo mayo de 2009, ocurre algo muy similar. El 68,5\% de los artículos relacionados con drogas se publicaron en páginas pares, mientras que solo el 1,5\% lo hicieron en la portada. Estos datos dan una idea de que el tema "drogas" no es tratado, por su ubicación, como un tema importante en las publicaciones estudiadas.

Si atendemos a la forma de aparición de los textos analizados, en el periodo abril-junio de 2008, la mayor parte de las informaciones se dedican totalmente al tema de las drogas (66,5\%) y, en el caso de ser un tema secundario, aparece bastante destacado como incrustación más fotografía (17,5\%).En el caso del periodo mayo de 2009, la mayoría de los artículos también se dedican totalmente al tema de las drogas (21\%). Sin embargo, en este periodo, cuando el tema es secundario, no aparece muy destacado, sino como referencia $(18 \%)$.

Si hacemos referencia al género de los textos, en el periodo abril-junio de 2008, predomina el informativo (noticias y fotonoticias, 56\%) y también los interpretativos (reportajes y entrevistas, 36,5\%).En el periodo 
mayo de 2009, aumenta el porcentaje de los artículos interpretativos (55\%), que predominan junto a los informativos (31,5\%).En muy pocas noticias se aborda el consumo de drogas. Solo encontramos un reportaje en profundidad en la revista Nuevo Vale sobre los peligros del consumo de los diferentes tipos de sustancias adictivas, aunque excluye el alcohol, lo que redunda en que dicha sustancia sea proyectada a sus lectores como "no droga", siendo justamente la audiencia de estas revistas el público diana sobre el que llaman la atención las autoridades por el inicio temprano ("botellón”) y mantenido en el consumo de alcohol.

Dentro de la intensidad formal de los textos, también hemos analizado el acompañamiento gráfico. En el periodo abril-junio de 2008, existe un uso importante de recursos gráficos (94\% de los artículos tienen recursos gráficos) si bien, es el formato habitual en el tratamiento del conjunto de las noticias de estas publicaciones. El 62,5\% del total estaban acompañados de una fotografía. En el periodo mayo de 2009, los resultados son similares. Un $88 \%$ de los artículos tienen acompañamiento gráfico. De ellos, destacan los que llevan una fotografía ( $51 \%$ del total). La intensidad formal de la información favorece, en general, que la noticia no pase desapercibida puesto que, aunque la ubicación suele ser en página par, se hace uso habitual de recursos gráficos y predominan los géneros interpretativos o informativos acompañados de recursos gráficos (fotonoticia). Todo ello propicia que se trate de informaciones que captan mucha atención.

\subsubsection{Resultados sobre el tono de la información}

La mayoría de los artículos relacionados con drogas y publicados en el periodo abril-junio de 2008 (82\%) realiza un tratamiento neutro del tema: dan el dato o hecho noticiable (por ejemplo, el artículo titulado "Drogas, pastis, coca, tripis... Informate" en la Revista Nuevo Vale (número 1553). No obstante, también hemos encontrado un número importante de artículos (9,5\%) donde se frivoliza (un ejemplo podría ser el bocadillo del artículo "Tanto monta” de la sección Actualidad de la Revista Cuore pronunciado ficticiamente por Pete Doherty y una acompañante (conocido por su aficción a las drogas): Chuf, Chuf, Chuf, ¡Qué bien sienta el cigarrito! ¡Chuf, Chuf, Chuf y empalmo con el siguiente). "En el periodo mayo de 2009, en un 77,5\% de los artículos se trata el tema de forma neutra y en un 18\% de ellos se frivoliza (por ejemplo, el artículo "El beso de la muerte" donde encontramos en bocadillos y en el texto fragmentos como: "a mí que me inviten a copas y lo demás da igual...” y “primero corrió el champán, después las fresas y luego el tabaco...”).

Además, llama poderosamente la atención que solo un 6,5\% y un 1,5\% de los artículos publicados sobre el consumo de drogas en abril-junio de 2008 y en mayo de 2009 respectivamente mantengan posturas en contra del consumo. También llama la atención que la revista Loka, en el periodo abril-junio de 2008, tenga un índice de artículos que frivolizan sobre las drogas de un 28,5\%, muy por encima de la media, y en el periodo mayo de 2009 , de un $60 \%$. 
Si se relacionan las sustancias tratadas en los textos con el tono, en abril-junio de 2008, la mayoría de los artículos que explicitan la sustancia tienen tono neutro (37,5\%), pero también hay gran número de artículos de tono frívolo (28,5\%) o en contra (27\%). En los artículos donde aparece el alcohol, el tono es frívolo en su mayoría (43,5\%), aunque también hay un amplio número de artículos neutros (37,5\%). En los artículos sobre tabaco, hay más que son de tono a favor (20\%) que en contra (10\%). Además, en los artículos sobre marihuana, el 66,5\% son de tono frívolo. Por ejemplo, en la Revista Nuevo Vale, en el número 1496 el artículo “!Ups!. Estoy un poco pi-piripi”.

En el periodo mayo de 2009, la mayoría de los artículos que explicitan la sustancia están tratados con tono neutro $(64,5 \%)$, pero también hay gran número de artículos de tono frívolo (27,5\%). En los artículos donde aparece el alcohol, hay un alto porcentaje que son de tono frívolo (37\%), aunque el tono neutro es mayoría (52,5\%). Un ejemplo de tono frívolo es el artículo "Un amor que continua” publicado en el número 158 de la Revista Cuore donde encontramos un bocadillo donde la actriz Elsa Pataky dice ficticiamente: "qué resaca tengo de la cenita de ayer...”.

En los artículos sobre tabaco, hay más que son de tono a favor (20\%) que en contra (0\%). Además, en los artículos sobre cocaína, el 66,5\% son de tono neutro (por ejmplo, el artículo "La cocaína y el cannabis en el aire que respiras" de la Revista Nuevo Vale (número 1555) y el 33,5\% son de tono frívolo.

Si tenemos en cuenta el papel de los medios de comunicación como orientadores de la opinión pública, que la gran mayoría de los artículos no se posicionen en contra de las drogas (93,5\% en el periodo abril-junio de 2008 y 98,5\% en el periodo mayo de 2009) lo que nos alerta sobre el escaso papel educativo o de prevención que están realizando las cabeceras estudiadas que, según los estudios españoles e internacionales, tienen un índice de lectura elevado ${ }^{7}$ entre el público que comienza a consumir drogas ${ }^{8}$.

En el análisis realizado en 2008, las sustancias con un mayor tratamiento son el tabaco y el alcohol, llamándonos la atención la no asociación de dichas sustancias con las drogas. En el caso de la marihuana, aunque el tratamiento es menor, se frivoliza en la mayor parte de las informaciones, lo que nos permite

\footnotetext{
Según el EGM, el diario de información general más leído, El País, tiene un índice de 2.081.000 lectores/día, seguido de El Mundo, con 1.309.000 lectores/día. Si pasamos a cabeceras regionales, en la Comunidad Valenciana, los dos diarios regionales más importantes, Levante y Las Provincias, tienen índices de 274.000 lectores/día y 167.000 lectores/día respectivamente. La revista más leída, Cuore, tiene un índice de lectura 2,95 veces superior al de Levante y 4,84 veces superior al de Las Provincias.

8 Según datos facilitados por la Asociación Valenciana de Consumidores y Usuarios (AVACU) de una encuesta con una muestra de 555 individuos de la Comunidad Valenciana, escolares de ambos sexos de 9 a 17 años, un 57,22\% de la muestra reconoce leer este tipo de revistas, frente a un $42,78 \%$ de afirma no leerlas. Por sexos, el 78,53\% son chicas y el 38,09\%, chicos. Además, cerca del $12 \%$ de los menores de edad reconoce leer revistas juveniles no recomendadas a menores.
} 
concluir que estas tres sustancias son proyectadas como "no drogas" o "drogas menores". Esta conclusión se repite en el estudio de seguimiento efectuado en 2009, en particular, en relación con el tratamiento del alcohol.

\section{Conclusiones}

La cobertura obtenida por el tema en ambos periodos objeto de estudio es, en general reducida. El número de informaciones es bajo y, en general, el fenómeno de las drogas es tratado en las revistas para adolescentes de manera poco explícita, encontrando además, referencias veladas en numerosos artículos donde aparecen personajes en situaciones de consumo.

Por consiguiente, los resultados obtenidos refutan claramente la hipótesis planteada por la que se esperaba encontrar un peso importante de esta temática en la prensa dirigida a adolescentes con una especial atención a prevenir el consumo de drogas en los medios dirigidos al público diana.

Igualmente los resultados de la investigación refutan la segunda hipótesis, según la cual preveíamos hallar una actitud no neutral frente a los efectos nocivos de la drogadicción, con un contexto y encuadre o frame de las informaciones que ayude a fomentar valores preventivos potenciando ejemplos positivos. Por el contrario, la mayoría de los textos analizados tienen como frame central el mundo de los famosos y la moda asociado a la drogadicción y no se halla ni un solo texto en el que el tema central sean las campañas o acciones positivas de ciudadanos o instituciones. Además, hemos constatado que estas revistas apenas dedican espacio para noticias y reportajes, que permitirían tratar el tema con rigor y centrar la atención del lector aportándole una información completa que le ayude a conocer las consecuencias del consumo de sustancias adictivas.

Además, en un porcentaje elevado de los artículos sobre el alcohol, las sustancias no son catalogadas como drogas. En muy pocos textos se aborda el consumo y, en los que lo hace, se trata con poco rigor, sobre todo teniendo en cuenta que las revistas analizadas son de consumo masivo entre la población juvenil que se encuentra en la franja de edad de inicio del consumo de drogas.

Asimismo, aunque la media de la intensidad formal de los textos no es muy elevada, el tema, la sección y el acompañamiento gráfico favorecen, en general, que estos no pasen desapercibidos para el lector.

La sección en la que se ubican mayoritariamente los artículos que están relacionados con las drogas, sección sobre famosos, y el tono neutro o frívolo predominante en estos textos contribuye a banalizar el problema y a la asociación de éxito con drogadicción. 
En referencia al tratamiento de los personajes, tal y como se planteaba en la tercera hipótesis, hay una especial atención a los famosos, pero igualmente queda refutada, porque los que más veces aparecen en las publicaciones analizadas en los ámbitos de Moda, Cine y Famosos, también se relacionan de manera significativa con el consumo de sustancias adictivas, con lo que se contribuye al fenómeno de la "glamurización" que aconseja evitar el departamento norteamericano Substance Abuse and Mental Health Service Administration y del que se hace eco el Plan Nacional sobre Drogas (Plan Nacional sobre Drogas, 2000: 27-28).

Otras conclusiones de la investigación son: al contrario de las recomendaciones de la Fundación de Ayuda para la Drogadicción sobre una información eficaz en materia de prevención, que incidían en evitar el trato de favor que se dispensa a determinadas drogas como las de síntesis, presentadas como inocuas, encontramos que, justamente las sustancias más tratadas, tabaco y alcohol, no son catalogadas como drogas en la mayoría de los textos. En el caso de la marihuana, se frivoliza en la mayor parte de los textos, lo que contribuye a proyectar estas sustancias como "no drogas" o "drogas blandas".

A partir de estos datos podemos concluir que esta familia de medios de comunicación no cumple la función social que se le supone para incrementar la percepción social del riesgo para la salud del consumo de drogas. No obstante, consideramos que esta familia de soportes debería ser considerada más desde las instituciones que trabajan en materia de prevención para mejorar los modelos informativos en relación al tema propiciados por dichas revistas, medios especialmente afines al público diana con una elevada capacidad de prescripción de estilos de vida.

Además, entendemos que el establecimiento de programas de trabajo conjuntos entre revistas e instituciones podría contribuir a una mayor difusión de modelos positivos y orientados a la prevención desde dichos soportes.

\section{Bibliografía}

Arza, J. (2002): Las drogas. Princesas y dragones. Madrid: Ediciones Eneida.

Bardin, J. L. (1986): El análisis de contenido. Madrid: Ediciones Akal.

Bauer, M.; Gaskell, G. (2002): Biotechnology. The making of a global controversy. Cambridge: Cambridge University Press.

Berkowitz, D. (1992): "Who sets the Media Agenda? The Ability of Policymakers to Determine News Decisions", en Kennamer, J.D. (ed.): Public Opinion, the press and public policy. London: Praeger, pp. 81-102.

Berrio, J. L. (2000): "Medios de comunicación y drogas", en Arana, X.; Vega Fuente, A.; Markez Alonso, I. (eds.): Drogas: cambios sociales y legales ante el tercer milenio. Madrid: Dykinson, pp. 219-234. 
Calafat, A.; PND. (2004): La diversión sin drogas: utopía y realidad. Palma de Mallorca: IREFREA España.

Cobb, R.W.; Elder, C.D. (1971): "The Politics of Agenda Building: An Alternative Perspective for Modern Democratic Theory", Journal of Politics, 33(4), pp. 892-915.

Colectivo Abierto de Sociología (1997): Tratamiento periodístico de las drogas y las drogodependencias. Madrid: Coordinadora de ONGs que Intervienen en Drogodependencias.

Costa, P. D.; Pérez, J.M. (1987): "La información sobre droga en la televisión en España", Comunidad y Drogas, 14, 31-45.

Costa, P. O.; Pérez, J. M. (1989): "Droga, televisión y sociedad", Comunidad y Drogas, 8.

Curtin, P. A. (1999): "Reevaluating public relations information subsidies: Market-driven journalism and agenda-building theory", Journal of Public Relations Research, 11(1), 53-90.

Del Pueyo, B. (2004): "Retos de la Información sobre Drogas en el siglo XXI ", en Hombre, A. P. (ed.): Desafíos y avances en la prevención y tratamiento de las drogodependencias. Madrid: Proyecto Hombre.

Del Río, P. (1991): "La información sobre problemas sociales en España. Medios de comunicación, el problema drogas y la percepción de soluciones", Comunidad y Drogas, 14, 59-99.

Diezhandino, M. P. (1994): El quehacer informativo. Bilbao: Universidad del País Vasco.

Driedger, M. (2008): "Creating shared realities through communication: exploring the agenda-building role of the media and its sources in the E. coli contamination of a Canadian public drinking water supply", Journal of Risk Research, 11, 23-40.

EMCDDA (2008): 2008 Annual report: the state of the drugs problem in Europe. Lisbon: European Monitoring Centre for Drugs an Drug Addiction.

EMCDDA (2009): Informe anual: El problema de la drogodependencia en Europa. Lisboa: Observatorio Europeo de las Drogas y Toxicomanías.

Entman, R. (1993): "Framing: Toward clarification of a fractured paradigm", Journal of Communication, 43(4), 51-58.

FAD (1998): Drogas: Conocer y Prevenir. Madrid: Venali S.A. Ediciones.

Fernández-Cid, M. (1996): Tratamiento periodístico de las drogas y las drogodependencia. Madrid: Coordinadoras de ONGs que intervienen en Drogodependencias.

Ferrer, X.; Dacosta, L. M. (1994): "Droga et mass-médias en Espagne. Problémes et recommendations", Psycotropes, 3, 103-105.

Fundación de Ayuda contra la Drogadicción (1998): Drogas: Conocer y Prevenir. Madrid: Venali, S.A. Ediciones.

Fundación de Ayuda contra la Drogadicción (2008): I Foro, Jóvenes, Drogas y Comunicación. Madrid: FAD.

Galdón, G. (1999): La enseñanza del Periodismo. Una propuesta de futuro. Barcelona: CIMS. 
Gamson, W. A.; Lash, K. E. (1983): "The political culture of social welfare policy", en Spiro, S.E.; Yuchman-Yaar, E. (eds.): Evaluating the welfare state: Social and political perspectives. New York: Academic Press, pp. 397-416.

Gamson, W.A.; Modigliani, A. (1989): "Media discourse and public opinion on nuclear power: A constructionist approach", American Journal of Sociology, 95(1), 1-37.

García Nebreda, B. (1987): La imagen de la droga en la prensa española. Madrid: Ministerio de Sanidad y Consumo.

Goffman, E. (1974): Frame analysis. An essay on the organization of Experience. Massachusets: Harvard University Press.

Goffman, E. (1981): Forms of talk. Philadelphia: University of Pennsylvania Press.

Kiousis, S.; Popescu, C.; Mitrook, M. (2007): "Understanding Influence on Corporate Reputation: An examination of Public Relation Efforts, Media Coverage, Public Opinion, and Financial Performance From Agenda-Building and Agenda-Setting", Journal of Public Relations Research, 19(2), pp. 147-165.

López Aranguren, E. (1989): "El análisis de contenido", en García Ferrando, M.; Ibáñez, J.; Alvira, F. (eds.): El análisis de la realidad social. Madrid: Alianza, pp. 383-414.

López de Luzuriaga, U.; Bermejo, S. (2004): "Qué esperamos los profesionales que tenemos que ver con las drogas de los mdc: la visión de una asociación de usuarios de drogas por la reducción de riesgos", en L. Pantoja, L.; Abeijón, J.A. (eds.): Los medios de comunicación y el consumo de drogas. Bilbao: Asociación Ai laket!, pp. 110-112.

Lowry, D.T.; Xie, L. (2007): Agenda-setting and Framing by Topic Proximity: A New Technique for the Computerized Content Analysis of Network TV News Presidential Campaign Coverage. Paper presented at the Political Communication Division of the International Communication Association, Annual Convention, San Francisco.

Martín Nieto, J. (1998): "Drogas de síntesis y medios de comunicación", Claves de Razón Práctica, 87, pp. 68-70.

Martínez Verdú, R. (2007): "Drogas, adolescentes y medios de comunicación", en Álvarez Pousa, L.; Evans Pim, J.; Crespo Argibay, Ó. (eds.): Comunicación e xuventude:Actas do Foro Internacional. Colexio Profesional de Xornalistas de Galicia, pp. 51-70.

McCombs, M. (2005). "A Look at Agenda-setting: past, present and future", Journalism Studies, 4, pp. 543-557.

Ministerio de Sanidad (2000): Actuar es posible: Medios de comunicación y drogodependencias. Madrid: Ministerio de Sanidad.

Oliva, M.P. (1986). "Los medios de comunicación social ante las drogodependencias". Comunidad y Drogas, 1, pp. 65-73.

Pantoja Vargas, L.; Abeijón, J. A. (2004): Los medios de comunicación y el consumo de drogas. Bilbao: Universidad de Deusto. Instituto Deusto de Drogodependencias.

Paricio Esteban, M. P. (1999): "Una aproximación a las dimensiones comunicativas de la moda: análisis de la comunicación de la moda en prensa de información general española durante el siglo XX", en VI Jornadas Internacionales de Jóvenes Investigadores en Comunicación: libro de actas.Valencia (España) del 19 al 23 de abril de 1999. Barcelona: Asociación Internacional de Jóvenes Investigadores en Comunicación, pp. 53-60. 
Paricio Esteban, P.; Sanfeliu Aguilar, P.; Sanfeliu Montoro, A. (2002): "Las campañas de comunicación y publicitarias sobre sida y drogas", Revista española de drogodependencias, 27(3), pp. 489-513.

Partenen, J.; Montoenm, M. (1989): El alcohol y los medios de comunicación. Madrid: Ministerio de Sanidad.

Plan Nacional sobre Drogas (2000): Medios de Comunicación y Drogodependencias. Madrid: Ministerio del Interior.

Prieto, J. L. (1987): "La imagen de la droga en la prensa española", Comunidad y Drogas, 4.

Quintá, F. (1989): "La tentadora evasión: medios de comunicación y drogas", Derechos Humanos: revista de la Asamblea Permanente por los Derechos Humanos, 22, pp. 31-32.

Rekalde, Á., y Romaní, O. (2002): Los medios de comunicación social ante el fenómeno de las drogas: un análisis crítico. Vitoria-Gasteiz: Servicio Central de Publicaciones del Gobierno Vasco.

Romero, F. J.; Carcelén, R. (2010): "El consumo de drogas en los jóvenes", en ediciones, E. (ed.): Campañas y comunicación institucional para la prevención de la drogadicción. Villafranca del Penedés: Erasmus ediciones.

Sádaba, M. T. (2001): "Origen, aplicación y límites de la Teoría del Encuadre en Comunicación", Comunicación y Sociedad, 14(2), pp. 143-173.

Scheufele, D. A. (1999): "Framing as a theory of media effects", Journal of Communication, 49, pp. 101-120.

Shallot, L. M.; Johnson, E. A. (2006): "Investigating relationships between journalist and public relation practitioners: Working togheder to se, frame and build the public agenda, 1991-2004", Public Relations Review, 32, pp. 151-159.

Sitthi-amorn, C.; Ngamvithayapongse, J. (1998): "The role of media and communication in improving the use of drugs and other technologies", International Journal of Technology Assessment in Health Care, 14(1), pp. 71-80.

Solans, M. (1991): Medios de comunicación, el "problema drogas" y la percepción de soluciones. Madrid: Ministerio de Sanidad y Consumo.

Trammell, K.; Brown, C. (2007): The Most Precious Cargo": The Role of Information Subsidies in Attribute-Agenda Setting in Media Coverage of the Evacuation of U.S. Citizens from Lebanon. Paper presented at the International Communication Association.

Turk, J. (1986): "Information Subsidies and Media Content: A Study of Public Relations Influence on the News", Journalism Monographs, 100, pp. 1-29.

UNODC (2008): World Drug Report 2008. United Nations Office on Drugs and Crime.

UNODC (2010): World Drug Report 2010. United Nations Office on Drugs and Crime.

Vega Fuente, A. (1995): "Los medios de comunicación social y las drogas: entre la publicidad y el control social", Revista española de drogodependencias, 20(2), pp. 99-111.

Vega Fuente, A. (2004): "Las drogas y los medios de comunicación: ¿Prohibir o domesticar? Apuntes para una nueva enseñanza-aprendizaje", Enseñanza, 22, pp. 369-389. 